\title{
On a generalization of the Monkey and Coconuts Problem
}

\author{
Amitabha Tripathi \\ Department of Mathematics, Indian Institute of Technology \\ Hauz Khas, New Delhi, India \\ e-mail: atripath@maths.iitd.ac.in
}

Received: 29 February 2020 Revised: 24 October $2020 \quad$ Accepted: 4 November 2020

\begin{abstract}
The original version of the Monkey and Coconuts Problem describes a hypothetical situation where five sailors and a monkey are trapped on an island and plan on equally sharing coconuts. The number of coconuts turns out to be one more than a multiple of 5 . The first sailor tosses one coconut to the monkey, and then takes his equal share out of the pile. Each subsequent sailor finds the number of remaining coconuts to be one more than a multiple of 5 , and repeats this process one at a time. After the last sailor has tossed one coconut to the monkey, he too takes his share. The number of coconuts that remain at this stage is a multiple of 5 , and is shared equally by the five sailors. In a variation of the original problem, the number of coconuts that remain after the fifth sailor has had his share is again one more than a multiple of 5 . Therefore, one coconut is again tossed to the monkey before the remaining pile can be equally distributed among the five sailors. The problem is to determine the least number of coconuts, and the final share for each sailor in each version.

We find explicit solutions for both the original version and the variation in the general case of $n$ sailors in which at each stage $r$ coconuts are tossed to the monkey. Even more generally, we also investigate the two versions when the $n$ sailors leave $r_{1}, \ldots, r_{n}$ coconuts to the monkey.
\end{abstract}

Keywords: Linear Diophantine equations, Continued fractions.

2010 Mathematics Subject Classification: 00A08, 97A20.

\section{The problem}

The Monkey and Coconuts Problem is possibly one of the most well-known, and one of the most worked-on problem in recreational mathematics. The American mathematician Martin Gardner, 
best known for creating and sustaining general interest in recreational mathematics for a large part of the 20th century, principally through his Scientific American "Mathematical Games" columns from 1956 to 1981, ranks this problem among his favourites according to an interview in [2] with his son.

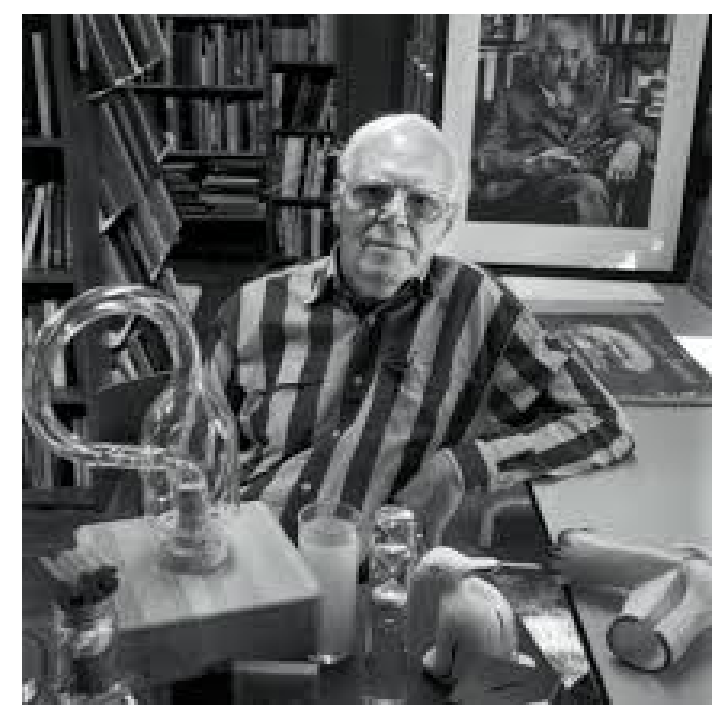

Figure 1. Martin Gardner (1914-2010)

Photo credit: Elliott Erwitt/Magnum Photos [2]

The problem is widely believed to trace its origin as "Coconuts" in a 1926 issue of The Saturday Evening Post by Ben Ames Williams [16].

Five men and a monkey were shipwrecked on a desert island, and they spent the first day gathering coconuts for food. Piled them all up together and then went to sleep for the night.

But when they were all asleep one man woke up, and he thought there might be a row about dividing the coconuts in the morning, so he decided to take his share. So he divided the coconuts into five piles. He had one coconut left over, and gave it to the monkey, and he hid his pile and put the rest back together.

By and by, the next man woke up and did the same thing. And he had one left over and he gave it to the monkey. And all five of the men did the same thing, one after the other; each one taking the fifth of the coconuts in the pile when he woke up, and each one having one left over for the monkey. And in the morning they divided what coconuts were left, and they came out in five equal shares. Of course each one must have known that there were coconuts missing; but each one was guilty as the others, so they didn't say anything. How many coconuts were there in the beginning?

However, Gardner in [6] writes that "Williams did not invent the coconut problem. He merely altered the problem to make it more confusing. The older version is the same except that in the morning, when the final division is made, there is again a final coconut for the monkey; in Williams's version, the division comes out even." 
A common method of solution to this problem is to formulate a linear Diophantine equation involving the number of coconuts and the equal share of each man in the morning, and then employ continued fractions to complete the solution. If $N$ denotes the number of coconuts, then it is easily verified that the five men leave

$$
\frac{4 N-4}{5}, \quad \frac{16 N-36}{25}, \quad \frac{64 N-244}{125}, \quad \frac{256 N-1476}{625}, \quad \frac{1024 N-8404}{3125}
$$

coconuts after taking their "share" at night. So if $a$ were to denote the number of coconuts received by each man in the morning, we have

$$
1024 N-15625 a=8404 .
$$

The continued fraction expansion for $\frac{15625}{1024}$ is $[15,3,1,6,2,1,3,2,1]$, as can be seen by repeated application of the division algorithm to compute the gcd. Hence the convergents to $\frac{15625}{1024}$ are

$$
\frac{15}{1}, \frac{46}{3}, \quad \frac{61}{4}, \quad \frac{412}{27}, \frac{885}{58}, \frac{1297}{85}, \frac{4776}{313}, \frac{10849}{711}, \frac{15625}{1024} .
$$

The penultimate convergent gives rise to a particular solution of eqn. (2):

$$
N_{0}=8404 \times 10849=91174996, \quad a_{0}=8404 \times 711=5975244 .
$$

The general solution of eqn. (2) is

$$
(N, a) \in\{(91174996+15625 t, 5975244+1024 t): t \in \mathbb{Z}\}=\{(3121+15625 t, 204+1024 t): t \in \mathbb{Z}\} .
$$

Thus the least number of coconuts is 3121 , and the corresponding share of coconuts for each man is 204 .

In order to solve the original problem, in which there is a coconut left over in the morning after dividing into five equal shares, we must have $\frac{1024 N-8404}{3125} \equiv 1(\bmod 5)$. Again, if $a$ were to denote the number of coconuts received by each man in the morning, we have

$$
1024 N-15625 a=11529 .
$$

The general solution of eqn. (3) is

$$
(N, a) \in\{(125078121+15625 t, 8197119+1024 t): t \in \mathbb{Z}\}=\{(15621+15625 t, 1023+1024 t): t \in \mathbb{Z}\} .
$$

Thus the least number of coconuts is 15621 , and the corresponding share of coconuts for each man is 1023 .

Both versions of the Monkey and Coconuts Problem have been source of many interesting articles, and have found a place in books [3, 5, 6, 10-12] and articles [1, 4, 7, 9, 13-15]. Kirchner [8] discusses a generalization of the problem in which there are $n$ sailors, with the first sailor discarding $r_{1}$ coconuts to make the division come out even and then taking his share. This process is repeated, with the $i$-th sailor discarding $r_{i}$ coconuts and then taking $1 / n$ of what remains. After the last sailor has taken his share, the sailors discard $r_{n+1}$ coconuts to make the division come out even and take an equal share of the remaining coconuts. Thus both versions of the problem correspond to the case where $n=5$ and $r_{i}=1$ for $1 \leq i \leq 5 ; r_{6}$ equals 1 in the original version and 0 in the Williams version. 


\section{The solutions}

We consider the following generalization to the Monkey and Coconuts Problem. We assume that there are $n$ sailors, and each discards a fixed number $r$ of coconuts to make the division come out even. We denote by $a_{k}$ the "share" of the $k$-th sailor at night, and by $a$ the common share of the sailors in the morning. For the original version of the problem, $r$ coconuts are discarded in the morning before the division comes out even whereas in the Williams version the coconuts may be divided into $n$ equal piles.

We use the argument above to find a solution to the generalized problems. For the case of $N$ coconuts and $n$ sailors, with $r$ coconuts left over at each stage, the number of coconuts left by the sailors follows the sequence

$\frac{n-1}{n}(N-r), \quad\left(\frac{n-1}{n}\right)^{2}(N-r)-\frac{n-1}{n} r, \quad\left(\frac{n-1}{n}\right)^{3}(N-r)-\left(\frac{n-1}{n}\right)^{2} r-\frac{n-1}{n} r, \quad \ldots$

The number of coconuts left by the $k$-th sailor is given by

$$
\left(\frac{n-1}{n}\right)^{k} N-\left(\frac{n-1}{n}+\left(\frac{n-1}{n}\right)^{2}+\cdots+\left(\frac{n-1}{n}\right)^{k}\right) r .
$$

When $k=n$, a little simplification yields

$$
\frac{(n-1)^{n} N-(n-1)\left(n^{n}-(n-1)^{n}\right) r}{n^{n}} .
$$

If each sailor receives $a$ coconuts in the morning, we have the equation

$$
(n-1)^{n} N-n^{n+1} a=(n-1)\left(n^{n}-(n-1)^{n}\right) r
$$

in the Williams version. Note that the special case $n=5$ and $r=1$ gives eqn. (2).

For the original version of the problem we are led to the equation

$$
(n-1)^{n} N-n^{n+1} a=\left(n^{n+1}-(n-1)^{n+1}\right) r .
$$

The special case $n=5$ and $r=1$ gives eqn. (3).

If we were to follow the methods adopted in the special cases, we would require the continued fraction expansion of $\frac{n^{n+1}}{(n-1)^{n}}$. While this is easily done for any specific value of $n$, such an expansion seems infeasible in general, as a function of $n$. However, there is a different, more direct and much simpler solution to eqn. (5) and eqn. (6).

Rearranging the terms in eqn. (5) and simplifying gives

$$
(n-1)^{n}(N+(n-1) r)=n^{n}(n a+(n-1) r) .
$$

Since $\operatorname{gcd}\left((n-1)^{n}, n^{n}\right)=1$, from eqn. (7) we have $n^{n} \mid(N+(n-1) r)$. Thus $N=\lambda n^{n}-(n-1) r$, $\lambda \in \mathbb{N}$. By substituting this value of $N$ in eqn. (7), $n a=\lambda(n-1)^{n}-(n-1) r, \lambda \in \mathbb{N}$. The last equation requires $\lambda \equiv(-1)^{n-1} r(\bmod n)$. We record the solution in

$$
N=\lambda n^{n}-(n-1) r, \quad a=\frac{\lambda(n-1)^{n}-(n-1) r}{n}, \quad \lambda \equiv(-1)^{n-1} r \quad(\bmod n), \lambda \in \mathbb{N} .
$$


Rearranging the terms in eqn. (6) and simplifying gives

$$
(n-1)^{n}(N+(n-1) r)=n^{n+1}(a+r) .
$$

Since $\operatorname{gcd}\left((n-1)^{n}, n^{n+1}\right)=1$, from eqn. (9) we have $n^{n+1} \mid(N+(n-1) r)$. Thus, $N=\lambda n^{n+1}-(n-1) r, \lambda \in \mathbb{N}$. By substituting this value of $N$ in eqn. (9), $a=\lambda(n-1)^{n}-r$. We record the solution in

$$
N=\lambda n^{n+1}-(n-1) r, \quad a=\lambda(n-1)^{n}-r, \quad \lambda \in \mathbb{N} .
$$

There is a slightly different approach to this generalization of the Monkey and Coconuts Problems. Instead of using the sequence of coconuts left over by the sailors as the starting point, we derive an expression for $N$ in terms of successive $a_{k}$ 's and $r$, ending with an expression for $N$ in terms of $a$ and $r$. We then use modular arithmetic to derive the expressions in eqn. (10) and eqn. (8).

The original problem may be formulated by the system of equations

$$
N-a_{1}-a_{2}-\cdots-a_{k-1}-(k-1) r=n a_{k}+r \quad(1 \leq k \leq n+1),
$$

where $a_{n+1}=a$. Taking the difference of any two successive equations, for $k \in\{1, \ldots, n+1\}$ we have

$\left(n a_{k}+r\right)-\left(n a_{k-1}+r\right)=\left(N-a_{1}-\cdots-a_{k-1}-(k-1) r\right)-\left(N-a_{1}-\cdots-a_{k-2}-(k-2) r\right)=-a_{k-1}-r$.

Thus, we have the simplified system of equations

$$
(n-1) a_{k-1}=n a_{k}+r \quad(1 \leq k \leq n+1),
$$

where $a_{n+1}=a$ as before. Starting with $N=n a_{1}+r$ and repeatedly using $a_{k-1}=\frac{n}{n-1} a_{k}+\frac{r}{n-1}$ leads to

$$
N=\frac{n^{n+1}}{(n-1)^{n}} a+\left(\frac{n^{n+1}-(n-1)^{n+1}}{(n-1)^{n}}\right) r .
$$

This is eqn. (6).

The solution we provided for both eqn. (5) and eqn. (6) was made possible by a happy coincidence of rearrangement of terms that led to a factorization and consequent solution. A more general method to solve these equations is by using congruences.

To solve eqn. (6), reduce modulo $n^{n+1}$ to get $(n-1)^{n} N \equiv-(n-1)^{n+1} r\left(\bmod n^{n+1}\right)$. Since $\operatorname{gcd}(n-1, n)=1, N \equiv-(n-1) r\left(\bmod n^{n+1}\right)$, so that $N=\lambda n^{n+1}-(n-1) r, \lambda \in \mathbb{N}$. Substituting in eqn. (6), we get $a=\lambda(n-1)^{n}-r, \lambda \in \mathbb{N}$. This is eqn. (10).

Williams's version of the problem is formulated by eqn. (11) and eqn. (12), with the righthand side of last equation (corresponding to $k=n+1$ ) in each having $r=0$. As in the case of the original version, starting with $N=n a_{1}+r$ and repeatedly using $a_{k-1}=\frac{n}{n-1} a_{k}+\frac{r}{n-1}$ leads to eqn. (5).

To solve equation eqn. (5), reduce modulo $n^{n}$ to get $(n-1)^{n} N \equiv-(n-1)^{n+1} r\left(\bmod n^{n}\right)$. Since $\operatorname{gcd}(n-1, n)=1, N \equiv-(n-1) r\left(\bmod n^{n}\right)$, so that $N=\lambda n^{n}-(n-1) r, \lambda \in \mathbb{N}$. Substituting in eqn. (6), we get $n a+(n-1) r=\lambda(n-1)^{n}, \lambda \in \mathbb{N}$. Since $\operatorname{gcd}(n-1, n)=1$, we have $(n-1) \mid a$. Substitute $a=(n-1) b$ in the last equation and divide through by $n-1$ to get $\lambda \equiv(-1)^{n-1} r(\bmod n)$. This is eqn. (8). 


\section{Remarks on a further generalization}

The generalization of the Monkey and Coconuts Problem that we have discussed thus far lends itself to an obvious extension, that of non-uniformity in the number of coconuts to be thrown away by each sailor. Thus, we may assume that the $k$-th sailor needs to throw away $r_{k}$ coconuts in order to be able to equally divide the pile of coconuts into $n$ parts of $a_{k}$ coconuts. This is the problem discussed by Kirchner [8]; our discussion is the special case where $r_{k}=r$ for $1 \leq k \leq n$. Further, we have $r_{n+1}=r$ in the original version and $r_{n+1}=0$ in the Williams version. This generalization does not lead to a neat solution, as we shall presently see.

This generalized problem may be formulated by the system of equations

$$
N-\left(a_{1}+\cdots+a_{k-1}\right)-\left(r_{1}+\cdots+r_{k-1}\right)=n a_{k}+r_{k} \quad(1 \leq k \leq n+1),
$$

and by taking the difference of any two successive equations, for $k \in\{1, \ldots, n+1\}$, the simplified system of equations

$$
(n-1) a_{k-1}=n a_{k}+r_{k} \quad(1 \leq k \leq n+1) .
$$

Starting with $N=n a_{1}+r_{1}$ and repeatedly using $a_{k-1}=\frac{n}{n-1} a_{k}+\frac{r_{k}}{n-1}$ leads to

$$
N=\frac{n^{n+1}}{(n-1)^{n}} a+\sum_{k=1}^{n+1}\left(\frac{n}{n-1}\right)^{k-1} r_{k}
$$

or to

$$
(n-1)^{n} N-n^{n+1} a=n^{n} r_{n+1}+n^{n-1}(n-1) r_{n}+\cdots+(n-1)^{n} r_{1} .
$$

The special case $r_{k}=r$ for $k \in\{1, \ldots, n+1\}$ is eqn. (6) and the special case $r_{k}=r$ for $k \in\{1, \ldots, n\}, r_{n+1}=0$ is eqn. (5).

Eqn. (15) is a linear Diophantine equation in the variables $N$ and $a$, and can be solved by using the continued fraction expansion for $\frac{n^{n+1}}{(n-1)^{n}}$ but only when specific values of $r_{1}, \ldots, r_{n+1}$ are given. The methods that were used in the special cases we considered in this paper do not apply in this most general case. Kirchner [8] formulates the problem as a difference equation, and then provides a solution which is neither in closed form nor even computationally easy to evaluate, as is to be expected in this most general case.

We close by summarizing the results of this paper.

Theorem 3.1. Suppose the $n$ sailors toss away $r_{1}, \ldots, r_{n}$ coconuts in order to form $n$ equal piles of coconuts, and let $r_{n+1}$ denote the number of coconuts tossed away in the morning in order to again form $n$ equal piles of coconuts. Then the number $N$ of coconuts and the equal share a of coconuts left for each sailor satisfy the equation

$$
(n-1)^{n} N-n^{n+1} a=n^{n} r_{n+1}+n^{n-1}(n-1) r_{n}+\cdots+(n-1)^{n} r_{1} .
$$

In the original version, where $r_{k}=r$ for $k \in\{1, \ldots, n+1\}$, this equation reduces to

$$
(n-1)^{n} N-n^{n+1} a=\left(n^{n+1}-(n-1)^{n+1}\right) r,
$$

with solutions given by

$$
N=\lambda n^{n+1}-(n-1) r, \quad a=\lambda(n-1)^{n}-r, \quad \lambda \in \mathbb{N} .
$$


In the Williams version, where $r_{k}=r$ for $k \in\{1, \ldots, n\}$ and $r_{n+1}=0$, this equation reduces to $(n-1)^{n} N-n^{n+1} a=(n-1)\left(n^{n}-(n-1)^{n}\right) r$, with solutions given by

$$
N=\lambda n^{n}-(n-1) r, \quad a=\frac{\lambda(n-1)^{n}-(n-1) r}{n}, \quad \lambda \equiv(-1)^{n-1} r(\bmod n), \lambda \in \mathbb{N} .
$$

\section{References}

[1] Anning, N. (1951). Monkeys and Coconuts, The Mathematics Teacher, 54, 560-562.

[2] Antonick, G. (2013). Martin Gardner's The Monkey and the Coconuts, The New York Times, Available online: http://wordplay.blogs.nytimes.com/2013/10/ 07 /gardner-2/?\_r=0.

[3] Bowden, J. (1936). The Problem of the Dishonest Men, the Monkeys, and the Coconuts, Special Topics in Theoretical Arithmetic, Lancaster Press, 203-212.

[4] Brashear, P. W. (1967). Five Sailors and a Monkey, The Mathematics Teacher, 60, 597-599.

[5] Gardner, M. (1961). The Monkey and the Coconuts, Ch. 9 in The Second Scientific American Book of Puzzles \& Diversions: A New Selection, Simon and Schuster, 104-111.

[6] Gardner, M. (2001). The Colossal Book of Mathematics: Classic Puzzles, Paradoxes, and Problems, W. W. Norton \& Company, 3-8.

[7] Kung, S. (1998). More Coconuts, The College Mathematics Journal, 29, 312-313.

[8] Kirchner, R. B. (1960). The Generalized Coconut Problem, The American Mathematical Monthly, 67, 516-519.

[9] Moritz, R. E. (1928). Solution to Problem 3242, The American Mathematical Monthly, 35, $47-48$.

[10] Ogilvy, C. S., \& Anderson, J. T. (1988). Excursions in Number Theory, Dover, 52-54.

[11] Olds, C. D. (1963). Continued Fractions, Random House, 48-50.

[12] Pappas, T. (1989). The Monkey and the Coconuts, The Joy of Mathematics, Wide World Publishers/Tetra, pp. 226-227 and p. 234.

[13] Singh, S., \& Bhattacharya, D. (1997). On Dividing Coconuts: A Linear Diophantine Problem, The College Mathematics Journal, 29, 203-204.

[14] Shin, T., \& Salvatore, G. (1978). On Coconuts and Integrity, Crux Mathematicorum, 4, 182-185.

[15] Weisstein, E. W. (2020). Monkey and Coconut Problem, Mathworld Wolfram, Available online: http: / / mathworld.wolfram.com/MonkeyandCoconutProblem.html

[16] Williams, B. A. (1926). Coconuts, The Saturday Evening Post, Oct. 9, 1926. 